Brief article

\title{
Four-year-old Mandarin-speaking children's online comprehension of relative clauses
}

\author{
Wenchun Yang ${ }^{\mathrm{a}}$, Angel Chan ${ }^{\mathrm{a}, \mathrm{b}, \mathrm{c}, *}$, Franklin Chang ${ }^{\mathrm{d}, \mathrm{g}}$, Evan Kidd ${ }^{\mathrm{e}, \mathrm{f}, \mathrm{g}, * *}$ \\ ${ }^{\text {a }}$ The Hong Kong Polytechnic University, HKSAR, China \\ ${ }^{\mathrm{b}}$ The Hong Kong Polytechnic University - Peking University Research Centre on Chinese Linguistics, HKSAR, China \\ ${ }^{\mathrm{c}}$ Research Centre for Language, Cognition, and Neuroscience, HKSAR, China \\ ${ }^{\mathrm{d}}$ Kobe City University of Foreign Studies, Japan \\ ${ }^{\mathrm{e}}$ Max Planck Institute for Psycholinguistics, The Netherlands \\ ${ }^{\mathrm{f}}$ The Australian National University, Australia \\ ${ }^{\mathrm{g}}$ ARC Centre of Excellence for the Dynamics of Language, Australia
}

\section{A R T I C L E I N F O}

\section{Keywords:}

Mandarin

Children

Relative clauses

On-line processing

Permutation analysis

\begin{abstract}
A B S T R A C T
A core question in language acquisition is whether children's syntactic processing is experience-dependent and language-specific, or whether it is governed by abstract, universal syntactic machinery. We address this question by presenting corpus and on-line processing dat a from children learning Mandarin Chinese, a language that has been important in debates about the universality of parsing processes. The corpus data revealed that two different relative clause constructions in Mandarin are differentially used to modify syntactic subjects and objects. In the experiment, 4-year-old children's eye-movements were recorded as they listened to the two RC construction types (e.g., Can you pick up the pig that pushed the sheep?). A permutation analysis showed that children's ease of comprehension was closely aligned with the distributional frequencies, suggesting syntactic processing preferences are shaped by the input experience of these constructions.
\end{abstract}

\section{Introduction}

The processing of subject and object relative clauses (RCs) is a frequent topic of investigation in psycholinguistics because their study allows researchers to examine interactions between parsing procedures, input experience, and general memory processes. Chinese languages (e.g., Cantonese, Mandarin) are important in this literature because their typologically-rare combination of Subject-Verb-Object (SVO) word order and head-final RCs allows opposing theories of syntactic processing to be tested (e.g., Jäger, Chen, Li, Lin, \& Vasishth, 2015). Children's on-line processing of RCs is an important though understudied source of evidence in this debate; assuming a degree of continuity between children and adults, developmental data both constrain and extend the explanatory reach of parsing models.

In the current paper we investigated 4-year-old Mandarin-speaking children's processing of relative clauses (RCs). We take advantage of the fact that Mandarin has multiple RC constructions, as in (1)-(4).

(1) [RCmai3 wan2ju4]de ma1ma Subject-Extracted DE

buy toy de mother
'The mother that bought the toy'

(2) [RCma1ma mai3] de wan2ju4 object-Extracted DE mother buy de toy

'The toy that mother bought.'

(3) [RCmai3 wan2ju4]de na4 ge4 ma1ma Subject-Extracted DCL buy toy de that CL mother

'The mother that bought the toy'

(4) [RCma1ma mai3] de na4 ge4 wan2ju4 object-Extracted DCL mother buy de that CL toy

'The toy that mother bought'

Sentences (1) and (3) are subject-extracted RCs because the RC modifies the grammatical subject (the mother), whereas sentences (2) and (4) are object-extracted because the RC modifies the grammatical object. Crucially, in (1) and (2) the head noun, introduced by the nominaliser $d e$, is bare (henceforth 'DE-RCs'). In contrast, the head noun in (3) and (4) is introduced by the demonstrative (DEM) + classifier (CL) combination (henceforth 'DCL-RCs'). These two RC types have different discourse-functional properties (Chen, Ming, \& Jiang, 2015), and there is debate regarding their structural differences (Cheng \&

\footnotetext{
* Corresponding author at: Department of Chinese and Bilingual Studies, The Hong Kong Polytechnic University, HKSAR, China.

** Corresponding author at: Language Development Department, Max Planck Institute for Psycholinguistics, P.O. Box 310, 6500 XD Nijmegen, The Netherlands. E-mail addresses: angel.ws.chan@polyu.edu.hk (A. Chan), evan.kidd@mpi.nl (E. Kidd).
} 
Sybesma, 2009; Cheung \& Li, 2015), pointing to the conclusion that they constitute overlapping but partially-distinct constructions.

The typological uniqueness of Mandarin (SVO word order + headfinal RCs) allows the predictions of different parsing theories to be teased apart. Structurally-oriented approaches assume that that the parser privileges structural information, and that subject RCs should be easier to process because they are less structurally complex than object RCs (Friedmann, Belletti, \& Rizzi, 2009; Lin \& Bever, 2006). In contrast, Gibson's Dependence Locality Theory (DLT) predicts that processing load increases with the number of unresolved arguments in a sentence. This predicts an object-RC advantage for Mandarin RCs, because the linear distance between the head noun and the gap is shorter than that for subject RCs. Finally, experience-based approaches argue that the parsing choices depend on frequency information that is accumulated across the course of a speaker's developmental history with the language (e.g., Fitz, Chang, \& Christiansen, 2011; Hale, 2001; Levy, 2008; MacDonald, 2013; Vasishth, Chen, Li, \& Guo, 2013).

The weight of empirical evidence supports a subject RC advantage in Chinese (for a meta-analysis of adult data see Vasishth et al., 2013; for discussion of acquisition see Tsoi, Yang, Chan, \& Kidd, 2019). The data are consistent with both the structurally-oriented and the experience-based approaches, since the only structure that has been tested DE RCs - more frequently modify subjects than objects. ${ }^{1}$ However, corpus work has shown that DCL RCs have different distributional properties to DE RCs. Chen et al. (2015) reported that DCL RCs more frequently modified objects ( $70 \%$ vs $16 \%$ ), critically arguing that the DEM-CL sequence (i) signals the need to link an upcoming definite referent (i.e., the head noun) to a co-occurring referent within the RC, and (ii) most frequently this involves linking a grammatical object (i.e., the head noun) to the subject role within the RC. Thus the presence of the DEM-CL sequence is a probabilistically reliable cue to an object RC analysis.

The asymmetry in distribution of DCL- and DE-RCs is pertinent to theoretical debates concerning RC processing. Both the structural and linear-based accounts predict uniform processing of both RC construction types; the structural account predicts a subject advantage whereas the linear-based account predicts, all things being equal, an object advantage for Mandarin. However, experience-based accounts predict differential effects based on frequency-derived expectations. Thus testing the two structures in parallel provides a means with which to test the competing predictions of the different theoretical approaches. In the current research we: (i) present corpus data from child-directed speech to demonstrate that the two RC constructions differ in their distributional properties, and (ii) present the results of a visual world eye-tracking experiment that tested whether children's on-line parsing decisions are guided by this distributional information.

\section{Study 1: Corpus study of child directed speech}

We extracted all morphologically tagged adult utterances from six Mandarin child language corpora in CHILDES (approximately 380,000 words in total) using the LUCID Toolkit (Chang, 2017): AcadLang corpus (Zhou, 2019 doi:https://www.doi.org/10.21415/T5SC9D), Chang1 \& Chang 2 corpus (Chang, 1998), Tong corpus (Deng \& Yip, 2018) and Zhou 1 (Zhou, 2001) \& Zhou 2 (Li \& Zhou, 2004). Our target level of analysis was 'general RC-like' sequences (following Vasishth

\footnotetext{
${ }^{1}$ Note that the experience-based approach makes specific predictions about the location of the complexity effects on-line, whereas the structure-based approach does not. See Jäger et al. (2015) for more details. Note that the same authors also used the DEM-CL combination to introduce a RC, not a head noun, which has the effect of reducing a local syntactic ambiguity. Other adult processing studies (e.g. Lin \& Garnsey, 2011; Wu, Kaiser, \& Vasishth, 2018) also used DEM-CL before the RC. This structure differs from our DCL-RCs tested in terms of discourse-functional properties (Chen et al., 2015; Ming \& Chen, 2010).
}

Table 1

Frequencies of DE and DCL RC-like Sequences Attested in Child-Directed Speech.

\begin{tabular}{lll}
\hline & Subject RC-like & Object RC-like \\
\hline DE & 1456 & 568 \\
DCL & 4 & 33 \\
\hline
\end{tabular}

et al., 2013). These structures were 'RC-like because they had the same surface structure as Mandarin subject [V-N-de-(DEM)-(CL)-(N)] and object RCs [N-V-de-(DEM)-(CL)-(N)], but due to the plurifunctionality of $d e$ as a modification marker may not be RCs. Thus they include both genuine RCs and sentences that have the same surface structure. This is the relevant grain of frequency because the identity of the structure is not clear until the sentence-final head noun. Table 1 lists the structural frequencies of subject and object RC-like sequences for both DE and DCL structures.

Overall, DE RC-like sequences were far more frequent than DCL RClike sequences (2024 vs 37 tokens). Consistent with Vasishth et al.'s (2013) corpus study, subject RC-like DE sequences were 2.5 times more frequent than object-like DE RC sequences. For DCL RC-like sequences we see the opposite pattern: object RC-like sequences were 8.25 times more frequent than subject-like RC sequences.

The experience-based account predicts that children's parsing decisions will be predicted by corpus frequencies. This makes the unique prediction that the children will have a subject preference for DE RCs, but an object preference for DEM-CL RCs. In contrast, the competing theories make uniform predictions for the both structures. The structure-based approach predicts a subject advantage, under the assumption that universal parsing machinery prefers to relativise on subjects (Friedmann et al., 2009; Lin \& Bever, 2006; Wagers, Borja, \& Chung, 2018). In contrast, theories that conceptualize complexity based on linear distance, such as Gibson's (2000) DLT, predict an object advantage.

\section{Study 2: online study of developmental processing}

We tested the predictions of the three approaches in an experimental study assessing the online comprehension of DE and DCL RCs by young Mandarin-speaking children.

\subsection{Method}

\subsubsection{Participants}

Thirty-six monolingual Mandarin-speaking children aged from 4;3 to $4 ; 9$ (Mean $=4 ; 6, \mathrm{SD}=0 ; 1$ ) were recruited from a kindergarten in Beijing, China. Children were tested on both DCL and DE RC construction types in a within-subjects design. Since we were interested in children's online sentence processing when they correctly interpreted the RC, the children whose accuracy was too low to offer an accurate record of their eye movements were excluded. Following our similar work on Cantonese (Chan, Yang, Chang, \& Kidd, 2018), the inclusion criterion was set to $50 \%$ overall comprehension accuracy for each sentence condition. As such, thirteen children who did not score over $50 \%$ accuracy on both RC construction types were excluded. Two additional children did not score over the threshold for one RC construction type from both the DCL condition and DE RC conditions; their data was included for the condition in which they scored above the threshold. The final sample consisted of twenty-three children for each RC construction type. All participants were typically developing with no known language impairments (see supplementary materials S2 for comparison of included and excluded children).

\subsubsection{Materials}

Sixteen test sentences were constructed: eight in the DE condition and another eight in the DCL condition, with four subject RCs and four 


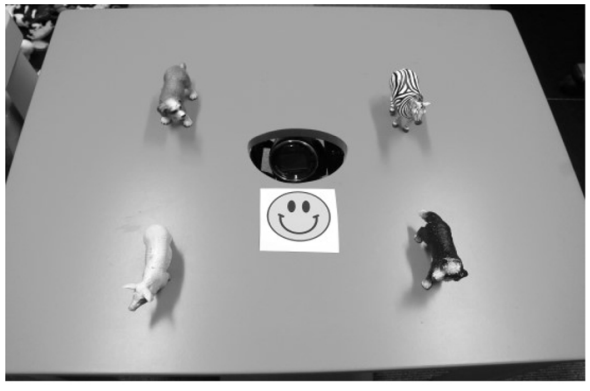

a

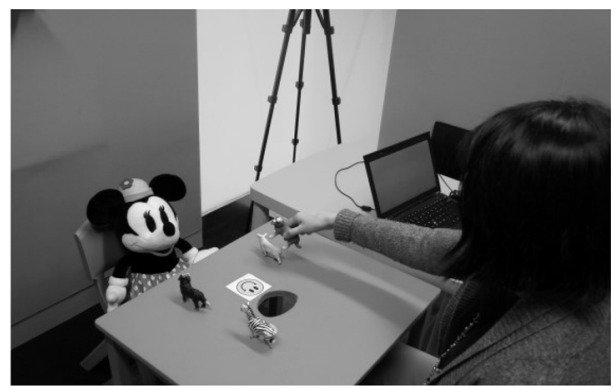

Fig. 1. (a) The layout of the toy props and the hidden digital camera to capture eye movements of the participant in the visual world eye-tracking task. (b) Experimenter acting out the pre-recorded background sentences (e.g., 'This dog is kicking the pig. The other dog is licking the pig') leading to the test sentence ('Can you pick up the dog that just kicked the pig?'). object RCs for each condition. The test sentences used animal names (e.g. dog, lion, zebra, bear, pig, monkey, cow, tiger, elephant, giraffe, horse, sheep, panda), and transitive verbs (e.g. chase, kick, wipe, tickle, lick, bump, bite, push, touch, feed) that are familiar to young children. All the sentences were pre-recorded by a female native Mandarin speaker (see Appendix A for the test sentences). A SONY HDR-CX580VE digital camera was used to record children's eye-movements.

\subsubsection{Procedure}

3.1.3.1. Referent selection task. The testing and data coding procedures followed those established in Chan et al. (2018). Four toy animals were placed on the table equidistant from a central camera (see Fig. 1a). In each case there were two tokens of the head referent (e.g., two dogs, as in sentence 5) and two additional toys that served other roles in the experimental items (i.e., as referents for the RC-internal noun, or the unrelated distractor). A smiley face sticker was placed at the centre of the table just below the camera. Before placing each animal toy on the table, the experimenter asked the child to name each animal character to ensure that the child knew their names.

Two background scenes consisting of one target scene [see (5a)] and one distractor scene [see $(5 \mathrm{~b})]$ were presented to provide a felicitous discourse context for using a restrictive RC (5c) (Corrêa, 1995; Hamburger \& Crain, 1982). The experimenter acted out each of the background scenes and then returned the animals back to their original positions before the next sentence played, as shown in Fig. 1b.

(5) a. Ni3 kan4! Zhe4 zhi1 xiao3gou3 zai4 ti1 zhe4 zhi1 xiao3zhu1 You look! This CL dog PROG kick this CL pig

'Look! This dog is kicking the pig.'

b. Yi2! Ling4wai4 yi1zhi1 xiao3gou3 zai4 tian3 zhe4zhi1 xiao3zhu1

Ooh! another one CL dog PROG lick this CL pig

'Ooh! The other dog is licking the pig.,

Attention getter:

Xian4zai4 qing3 kan4yi1xia4 zhong1jian1 na4 ge4 xiao4lian3

Now please look-at centre that CL smiley-face

'Now please look at the smiley face in the centre.'

c. Ni3 ke3-bu4-ke3yi3 na2qi3

You can-not-can pick-up

\# gang1cai2 ti1 xiao3zhu1 de na4 zhi1 xiao3gou3 ya

\# just-now kick pig de that CL dog SFP

'Can you pick up \# the dog that just kicked the pig?'

(\#: pause)

An attention getter ('now please look at the smiley face in the centre') was played after the background scenes, directing the children's attention away from the toys so that they were not biased by perceptual information when the test sentence began (e.g., by looking at one token of the head referent). A pause was added between the carrier phrase and the RC to avoid any garden path effects. Across trials

\footnotetext{
${ }^{2}$ Note: EXCL = exclamative (e.g., 'wow!'), PROG = progressive aspect marker.
}

the presentation of the target and the distractor animals in the background scenes was counterbalanced, and the location of the toys was pseudorandomized. From the child's perspective, the target and the distractor were put horizontally or diagonally, but never put along the same vertical plane, which served to facilitate the accurate coding of eye-movements. Two practice trials that did not contain RCs were used to familiarize children with the experimental procedure. The entire experiment lasted approximately 25 minutes per child.

3.1.3.2. Eye-movement coding. Children's eye movements were recorded by the camera placed under the table. The eye movements were coded frame-by-frame using the visual editing program Sound Forge $($ (Magix Software $\mathrm{GmbH}$ ). Looks to each of the four toys were recorded for each $40 \mathrm{~ms}$ frame. Coding started from the onset of the first syllable of the RC and continued until $2400 \mathrm{~ms}$ post RC onset. Data from six children $(27.3 \%$ of final sample) were re-coded by a second trained coder for inter-coder reliability across the two sentence conditions. The results suggested excellent reliability (DE: $r_{s}=.919$, $p<.001, k=.917$; DCL: $\left.r_{s}=.933, p<.001, k=.933\right)$.

\subsection{Results}

Children's offline accuracy was above chance ( $>60 \%$ for all conditions, where chance $=25 \%$ ), with no significant differences across conditions (see Supplementary Materials S3).

\subsubsection{Eye-movement data}

We only analysed eye-movement data for those trials during which children chose the correct referent. Fig. 2 depicts the proportion of looks to the target referent for DCL and DE RCs relative to the total looks to all four referents. To determine how the children's preferences changed as a result of hearing the sentence, we subtracted the target codes at the start of the RC from the rest of the target codes. For DE RCs there were more fixations to the target during subject in comparison to object RCs after the offset of the head noun. The DCL RCs showed the opposite pattern.

The data were analysed using a non-parametric permutation test, which follows a three-step procedure (see Chan et al., 2018 for a detailed tutorial). ${ }^{3}$ First, a series of linear regressions were conducted for every $40 \mathrm{~ms}$ time bin to predict looks to the target with extraction (subject vs. object) and RC construction type (DE vs. DCL) crossed. This yields the typical statistical information (e.g., beta coefficient, $p$-value) for the main effects of extraction and RC construction type, and an interaction for each time bin. We test here for the significance of the interaction because this is the most likely effect suggested by Fig. 2 as well as similar past research on Cantonese (Chan et al., 2018). These interaction $p$-values are depicted at -0.1 on the y-axis of Fig. 2; the

\footnotetext{
${ }^{3}$ For a justification of the use of the permutation test, and a comparison with other commonly used analytical approaches, see Supplementary Materials S4.
} 


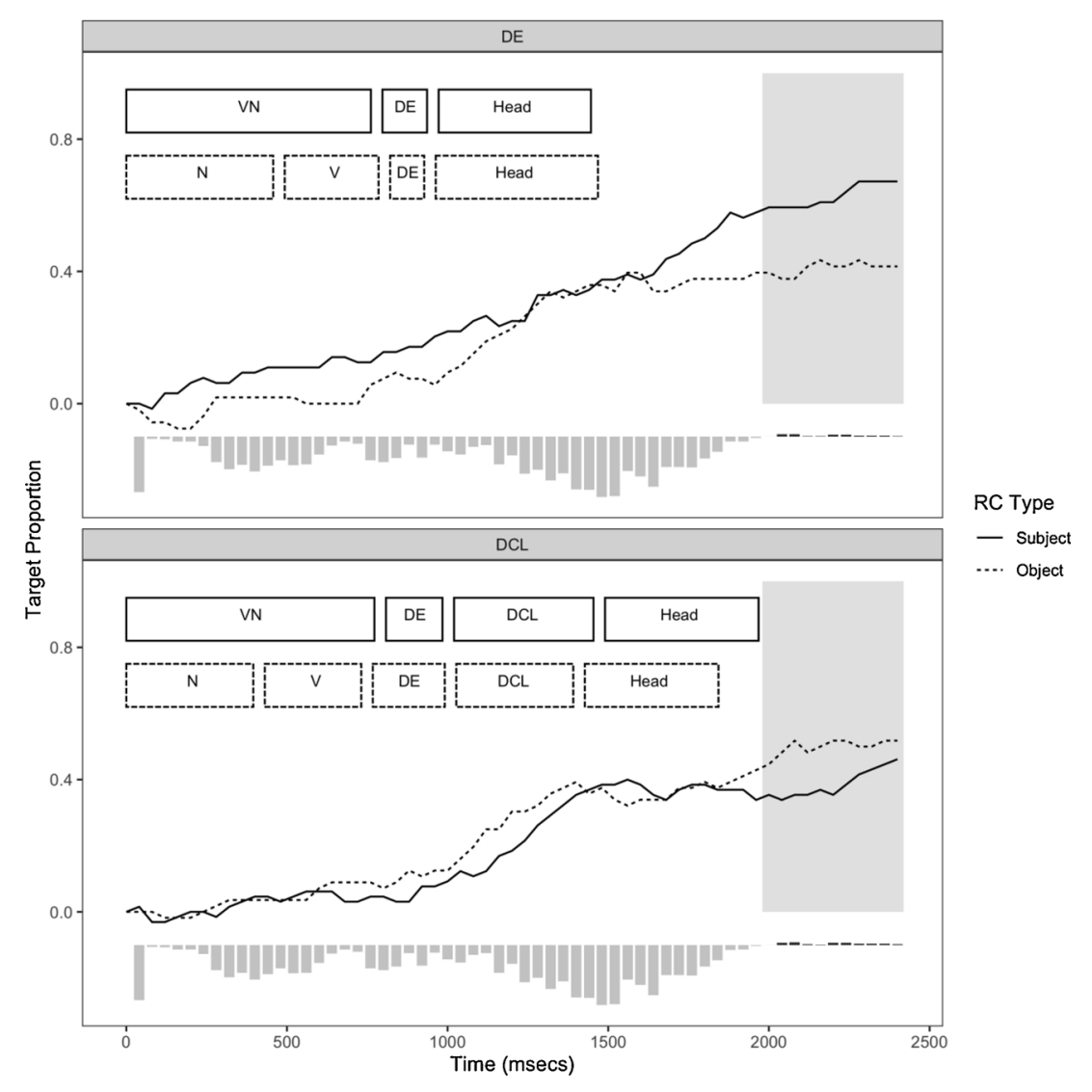

Fig. 2. Average target proportions of looks for the DE (top panel) and the DCL (bottom panel) RCs are shown by solid/ dashed lines. Onsets/offsets for different sentence units are shown by the size of the rectangles at the top left (solid for subject RCs, dashed for object RCs). Small grey/black bars near -0.1 are $p$-values for individual time bins. The large shaded grey bars represent the time-windows identified by the permutation analysis as significant. significant time bins are black bars that rise above -0.1. The next step was to cluster the time bins based on whether the interaction $p$-value term in adjacent time windows was less than 0.05 . This encodes the idea that effects in adjacent windows are not independent and hence should be treated as one processing effect. There is one cluster with significant interaction $p$-values starting from 2000 until 2400. The final step was to create a permutation distribution. To do this, we randomly permuted the extraction and RC construction type labels in the cluster, so that the original looking time is now randomly paired with extraction and RC construction type labels. We fit a regression model with extraction and RC construction type crossed on this randomized data and extracted the interaction $t$-value term. This procedure was repeated 1000 times, which yielded an exact distribution of sum $t$-values for the cluster, representing how likely this cluster would occur by chance. This revealed a significant interaction of extraction site and RC construction type for the cluster between $2000 \mathrm{~ms}$ and $2400 \mathrm{~ms}$ (sum $\mathrm{t}=22.224$, $p<.001$, shown as grey region on Fig. 2).

To understand this interaction, we created a separate permutation distribution for each RC construction type (DCL, DE). For DE RC constructions, subject extraction yielded more looks than object extraction in 2000-2400 ms window $(t=19.7, p<0.001)$; the opposite effect was observed for DCL RC constructions in the same window ( $t=11.5$, $p<0.001$ ). Overall, the permutation analysis yielded a single significant window, which provides clear evidence of an interaction of RC construction type and extraction site after the offset of the sentences in all conditions.

\section{Discussion}

Across two studies we found an asymmetry in the distribution of DE and DCL RC-like structures that matched 4-year-old monolingual children's on-line processing of subject- and object-extracted DE and DCL $\mathrm{RC}$ constructions. The result is consistent with experience-based accounts of parsing (e.g., Fitz et al., 2011; Levy, 2008; MacDonald, 2013; Vasishth et al., 2013), but is inconsistent with accounts that predict a uniform pattern of responding across both RC constructions (Diessel, 2007; Friedmann et al., 2009; Gibson, 2000; Lin \& Bever, 2006).

One question concerns which experience-based models are most compatible with the results? Many experience-based models use a preexisting grammar and a corpus tagged by adult linguists to assign probabilities to structures (e.g., Levy, 2008; Yun, Chen, Hunter, Whitman, \& Hale, 2015). These models would not be able to explain our results if they use a grammar that does not distinguish DE and DCL structures (e.g., as in Chen, Grove, \& Hale, 2012). There is evidence to suggest that the two structures are at least partially distinct (Chen et al., 2015; Cheung \& Li, 2015). Thus there are motivations for treating them differently, yet this can only happen if the experience-based model has an acquisition mechanism that can learn this distinction.

In contrast to models that implement existing grammars, connectionist models can learn typologically-different grammars (Hsiao \& MacDonald, 2013) and encode statistical information about discourse functions and sentence structures (e.g., given/new, Fitz \& Chang, 2017). These models also have production biases (Chang, 2009), which could provide an underlying basis for the frequency distribution that is encoded by the comprehension system (e.g., ERPs, Fitz \& Chang, 2019). This approach instantiates the Production-Distribution-Comprehension account (Gennari \& MacDonald, 2009; MacDonald, 2013), where frequency patterns in the input ultimately arise from ease of expressing different meanings within the constraints of the grammar and production system.

The current paper highlights the value of psycholinguistic research across lesser-studied languages and participant groups, particularly when those languages can help to distinguish competing theoretical predictions. Our study contains many of the limitations typical of developmental research, including the likelihood of large variability 
amongst participants (Kidd, Donnelly, \& Christiansen, 2018) and the use of fewer items and comparatively small sample sizes (Bergmann et al., 2018). Thus future research corroborating the findings is necessary to further gauge the role of the input in shaping the nature of language comprehension.

\section{Acknowledgements}

This research was supported by a research grant from the Hong Kong Polytechnic University to Chan (G-YBF9) and by an Australian Research Council grant to Kidd (CE140100041). We thank Silvia Gennari, Shravan Vasishth, and two anonymous reviewers for helpful comments. Annotated R code for our analyses can be found at https:// github.com/franklinr/PermutationAnalysis

\section{Appendix A}

Can you pick up [relative clause] head noun?

\section{Subject DE RCs}

1. 唋斑马 的扸子

tian3 ban1ma3 DE shi1zi

lick zebra DE lion

'the lion that licked the zebra'

2. 撞 狗熊的老虎

zhuang4 gou3xiong2 DE lao3hu3

bump bear DE tiger

'the tiger that bumped the bear'

3. 咬小牛的小象

yao3 xiao3niu2 DE xiao3xiang4

bite cow DE elephant

'the elephant that bit the cow'

4. 推长颈鹿的老虎

tui1 chang2jing3lu4 DE lao3hu3

push giraffe DE tiger

'the tiger that pushed the giraffe'

Object DE RCs

1. 熊猫舔的狮子

xiong2mao1 tian3 DE shi1zi

panda lick DE lion

'the lion that the panda licked'

2. 大象追的老虎

da4xiang4 zhui1DE lao3hu3

elephant chase DE tiger

'the tiger that the elephant chased'

3. 小猪踢的小牛

xiao3zhu1 ti1DE xiao3niu2

pig kick DE cow

'the cow that the pig kicked'

4. 大象 撞 的长颈鹿

da4xiang4 zhuang4 DE chang2jing3lu4

elephant bump DE giraffe

'the giraffe that the elephant bumped'

Subject DCL RCs

1. 追小獅子的那只小狗

zhui1 xiao3shi1zi DE na4 zhi1 xiao3gou3

chase lion DE that CL dog

'the dog that chased the lion'

2. 踢 斑马的那只狗熊

ti1 ban1ma3 DE na4 zhi1 gou3xiong2

kick zebra DE that CL bear

'the bear that kicked the zebra'

3. 擦小猪的那只小猴

ca1 xiao3zhu1 DE na4 zhi1 xiao3hou2

wipe pig DE that CL monkey

'the monkey that wiped the pig'
4. 挠小猴的那只小牛

nao2 xiao3hou2 DE na4 zhi1 xiao3niu2

tickle monkey DE that CL cow

'the cow that tickled the monkey'

Object DCL RCs

1. 小马 推的那只 小狗

xiao3ma3 tui1 DE na4 zhi1 xiao3gou3

horse push DE that CL dog

'the dog that the horse pushed'

2. 老虎咬的那只狗熊

lao3hu3 yao3 DE na4 zhi1 gou3xiong2

tiger bite DE that CL bear

'the bear that the tiger bit'

3. 小羊摸的那只小猴

xiao3yang2 mo1 DE na4 zhi1 xiao3hou2

sheep touch DE that CL monkey

'the monkey that the sheep touched'

4. 老虎喂的那只牛

lao3hu3 wei4 DE na4 zhi1 niu2

tiger feed DE that CL cow

'the cow that the tiger fed'

\section{Appendix B. Supplementary data}

Supplementary material related to this article can be found, in the online version, at https://doi.org/10.1016/j.cognition.2019.104103.

\section{References}

Bergmann, C., Tsuji, S., Piccinini, P. E., Lewis, M. L., Braginsky, M., Frank, M. C., et al. (2018). Promoting replicability in developmental research through meta-analyses: Insights from language acquisition research. Child Development, 89(6), 1996-2009. https://doi.org/10.1111/cdev.13079.

Chan, A., Yang, W. C., Chang, F., \& Kidd, E. (2018). Four-year-old Cantonese-speaking children's online processing of relative clauses: A permutation analysis. Journal of Child Language, 45(1), 174-203. https://doi.org/10.1017/S0305000917000198.

Chang, C. (1998). The development of autonomy in preschool Mandarin Chinesespeaking children's play narratives. Narrative Inquiry, 8(1), 77-111. https://doi.org/ $10.1075 /$ ni.8.1.05cha.

Chang, F. (2009). Learning to order words: A connectionist model of heavy NP shift and accessibility effects in Japanese and English. Journal of Memory and Language, 61, 374-397. https://doi.org/10.1016/j.jml.2009.07.006.

Chang, F. (2017). The LuCiD language researcher's toolkit [Computer software]. Retrieved fromhttp://gandalf.talkbank.org:8080/.

Chen, Z., Grove, K., \& Hale, J. (2012). Structural expectations in Chinese relative clause comprehension. Proceedings of the 29th West Coast Conference on Formal Linguistics (pp. 29-37).

Chen, L., Ming, T., \& Jiang, X. (2015). The relevance of the principle of relevance for word order variation in complex referring expressions in Mandarin Chinese. Lodz Papers in Pragmatics, 11(1), 77-104. https://doi.org/10.1515/lpp-2015-0005.

Cheng, L. S., \& Sybesma, R. (2009). De as an underspecified classifier: First explorations. Yuyanxue luncong [essays on linguistics], 39, Beijing: Shangwu Yinshuguan: The Commercial Press123-156.

Cheung, C. C. H., \& Li, H. Z. (2015). Inner and outer modifiers in Mandarin and Cantonese. Linguistic Sciences, 14, 449-458.

Corrêa, L. M. S. (1995). An alternative assessment of children's comprehension of relative clauses. Journal of Psycholinguistic Research, 24(3), 183-203.

Deng, X., \& Yip, V. (2018). A multimedia corpus of child Mandarin: The Tong corpus. Journal of Chinese Linguistics, 46(1), 69-92. https://doi.org/10.1353/jcl.2018.0002.

Diessel, H. (2007). A construction-based analysis of the acquisition of East Asian relative clauses. Studies in Second Language Acquisition, 29(2), 311-320. https://doi.org/10. $1017 /$ s0272263107070167.

Fitz, H., Chang, F., \& Christiansen, M. H. (2011). A connectionist account of the acquisition and processing of relative clauses. In E. Kidd (Ed.). The acquisition of relative clauses: Functional and typological perspectives (pp. 39-60). Amsterdam: John Benjamins.

Fitz, H., \& Chang, F. (2017). Meaningful questions: The acquisition of auxiliary inversion in a connectionist model of sentence production. Cognition, 166, 225-250. https:// doi.org/10.1016/j.cognition.2017.05.008.

Fitz, H., \& Chang, F. (2019). Language ERPs reflect learning through prediction error propagation. Cognitive Psychology, 111, 15-52. https://doi.org/10.1016/j.cogpsych. 2019.03.002.

Friedmann, N., Belletti, A., \& Rizzi, L. (2009). Relativized relatives: Types of intervention in the acquisition of A-bar dependencies. Lingua, 119(1), 67-88. https://doi.org/10. 1016/j.lingua.2008.09.002.

Gennari, S. P., \& MacDonald, M. C. (2009). Linking production and comprehension processes: The case of relative clauses. Cognition, 111(1), 1-23. https://doi.org/10. 
1016/j.cognition.2008.12.006

Gibson, E. (2000). The dependency locality theory: A distance-based theory of linguistic complexity. In Y. Miyashita, A. Marantz, \& W. O’Neil (Eds.). Image, language, brain (pp. 95-126). Cambridge, MA: MIT Press.

Hale, J. T. (2001). A probabilistic earley parser as a psycholinguistic model. Proceedings of the Second Meeting of the North American Chapter of the Association for Computational Linguistics.

Hamburger, H., \& Crain, S. (1982). Relative acquisition. In S. KuczajII (Ed.). Language development I (pp. 245-274). Hillsdale, NJ: Lawrence Erlbaum.

Hsiao, Y., \& MacDonald, M. C. (2013). Experience and generalization in a connectionist model of Mandarin Chinese relative clause processing. Frontiers in Psychology, 4, 767. https://doi.org/10.3389/fpsyg.2013.00767.

Jäger, L., Chen, Z., Li, Q., Lin, C. J. C., \& Vasishth, S. (2015). The subject-relative advantage in Chinese: Evidence for expectation-based processing. Journal of Memory and Language, 79, 97-120. https://doi.org/10.1016/j.jml.2014.10.005.

Kidd, E., Donnelly, S., \& Christiansen, M. H. (2018). Individual differences in language acquisition and processing. Trends in Cognitive Sciences, 22, 154-169. https://doi.org/ 10.1016/j.tics.2017.11.006.

Li, X. Y., \& Zhou, J. (2004). The effects of pragmatic skills of mothers with different education on children's pragmatic development. Master's thesis. Shanghai, China: Nanjing Normal University.

Lin, C. J. C., \& Bever, T. G. (2006). Subject preference in the processing of relative clauses in Chinese. In D. Baumer, D. Montero, \& M. Scanlon (Eds.). Proceedings of the 25th West Coast Conference on Formal Linguistics (pp. 254-260).

Lin, Y., \& Garnsey, S. (2011). Animacy and the resolution of temporary ambiguity in relative clause comprehension in Mandarin. In H. Yamashita, Y. Hirose, \& J. Packard (Eds.). Processing and Producing Head-Final Structures (pp. 241-275).

Levy, R. (2008). Expectation-based syntactic comprehension. Cognition, 106, 1126-1177. https://doi.org/10.1016/j.cognition.2007.05.006

MacDonald, M. C. (2013). Distributional information in language comprehension, production, and acquisition: Three puzzles and a moral. In B. MacWhinney (Ed.). The emergence of language (pp. 195-214). NY: Psychology Press.

Ming, T., \& Chen, L. (2010). A discourse-pragmatic study of the word order variation in Chinese relative clauses. Journal of Pragmatics, 42(1), 168-189. https://doi.org/10. 1016/j.pragma.2009.05.023.

Tsoi, E. Y. L., Yang, W., Chan, A., \& Kidd, E. (2019). Mandarin-English speaking bilingual and Mandarin speaking monolingual children's comprehension of relative clauses. Applied Psycholinguistics, 40(4), 933-964. https://doi-org.ezproxy.lb.polyu.edu.hk/ 10.1017/S0142716419000079.

Vasishth, S., Chen, Z., Li, Q., \& Guo, G. (2013). Processing Chinese relative clauses: Evidence for the subject-relative advantage. PloS One, 8(10), e77006. https://doi. org/10.1371/journal.pone.0077006.

Wagers, M. W., Borja, M. F., \& Chung, S. (2018). Grammatical licensing and relative clause parsing in a flexible word-order language. Cognition, 178, 207-221. https:// doi.org/10.1016/j.cognition.2018.05.006.

Wu, F., Kaiser, E., \& Vasishth, S. (2018). Effects of early cues on the processing of Chinese relative clauses: Evidence for experience-based theories. Cognitive Science, 42, 1101-1133. https://doi.org/10.1111/cogs.12551.

Yun, J., Chen, Z., Hunter, T., Whitman, J., \& Hale, J. (2015). Uncertainty in processing relative clauses across East Asian languages. Journal of East Asian Linguistics, 24(2), 113-148. https://doi.org/10.1007/s10831-014-9126-6.

Zhou, J. (2001). Pragmatic development of Mandarin speaking young children: From 14 months to 32 months. Unpublished doctoral dissertation. The University of Hong Kong.

Zhou, J. (2019). AcadLang Corpus. Retrieved fromhttps://doi.org/10.21415/ T5SC9Dhttps://childes.talkbank.org/access/Chinese/Mandarin/AcadLang.html. 